\title{
Gate-controlled conductance through bilayer graphene ribbons
}

\author{
J. W. González, ${ }^{1}$ H. Santos, ${ }^{2}$ E. Prada, ${ }^{2}$ L. Brey, ${ }^{2}$ and L. Chico ${ }^{2}$ \\ ${ }^{1}$ Departamento de Física, Universidad Técnica Federico Santa María, Casilla postal 110 V, Valparaíso, Chile \\ ${ }^{2}$ Instituto de Ciencia de Materiales de Madrid, (CSIC), Cantoblanco, 28049 Madrid, Spain \\ (Received 19 August 2010; revised manuscript received 2 March 2011; published 17 May 2011)
}

\begin{abstract}
We study the conductance of a biased bilayer graphene flake with monolayer nanoribbon contacts. We find that the transmission through the bilayer ribbon strongly depends on the applied bias between the two layers and on the relative position of the monolayer contacts. Besides the opening of an energy gap on the metallic bilayer, the bias allows to tuning the electronic density on the bilayer flake, making possible the control of the electronic transmission by an external parameter.
\end{abstract}

DOI: 10.1103/PhysRevB.83.205402

PACS number(s): 61.46.-w, 73.22.-f, 73.63.-b

\section{INTRODUCTION}

The experimental isolation of graphene layers has opened a new arena for the fundamental exploration of novel physical phenomena as well as the design of electronic nanodevices. The high mobility of carriers has motivated experiments where high-speed graphene-based field effect transistors have been demonstrated. ${ }^{1}$

However, the prospective use of graphene in nanoelectronics requires the possibility of opening gaps in its band structure in a controllable way. Because of the chiral nature of the carriers, ${ }^{2}$ it is not easy to open gaps and confine carriers in a single graphene monolayer. Carbon-based structures such as nanoribbons, ${ }^{3,4}$ nanotubes, ${ }^{5-7}$ and graphene bilayers ${ }^{8-13}$ are viable materials for nanoelectronics, since it is feasible to change their electronic characteristics from semiconducting to metallic as a function of geometric or external parameters. Bilayer graphene is a good candidate because a gap can be opened and controlled by an applied bias between its two layers. Theoretical predictions and optical measurements yield a gap value of around $200 \mathrm{meV}$ for bilayer graphene. ${ }^{8,9,14}$ However, electronic measurements give smaller gaps, probably because of the presence of gap states. The improvement in bilayer graphene fabrication could make possible the obtention of similar electronic and optical gaps in these systems. ${ }^{11,15,16}$

Monolayer graphene nanoribbons (MGNs) stand out as optimal electrodes for systems based on bilayer graphene, with the aim of achieving the best integration of nanoelectronic components. Narrow monolayer graphene nanoribbons have recently been obtained by different methods, ${ }^{17-19}$ and the observation of an electrically induced gap in bilayer graphene nanoribbons (BGNs) has been recently reported. ${ }^{15}$ The increasing success on the obtention of nanosized graphene strucutures points toward the feasibility of nanoribbon-based devices. Thus it is important to study the electronic transport of BGNs with MGN contacts. This system is the planar/graphene analog of telescoping carbon nanotubes, which were synthesized and experimentally characterized 10 years ago. ${ }^{20}$ Telescoping tubes have been used to construct an electromechanical resonator ${ }^{21}$ and have been proposed to build ultrafast nonvolatile memory devices. ${ }^{22}$ Monolayer graphene/bilayer graphene interfaces have been studied by other authors. ${ }^{8,23}$

Previous work on nanoribbons has focused on the electronic transport through bilayer graphene flakes in the absence of external gates. ${ }^{24}$ In such a case the conductance shows strong oscillations as a function of the energy of the incident electron and the length of the bilayer region. In this paper we show that the conductance of BGNs connected to MGNs strongly depends on the way the bilayer is contacted and on the applied gate voltage. This allows for an external control of the electronic properties of the system.

The article is organized as follows. In Sec. II we explain the geometries studied, i.e., the type of nanoribbons and electrode alignment, the models employed to describe the electronic properties of the different types of ribbons, and the two methods used to compute the conductance. We present our results in Sec. III. A summary is given in Sec. IV.

\section{GEOMETRIES, MODELS, AND METHODS}

\section{A. Geometries}

We analyze electronic transport in the linear regime through a gated BGN connected to two MGN contacts. The monolayer leads can be either armchair or zigzag graphene nanoribbons, ${ }^{3,4}$ serving as contacts to armchair or zigzag bilayer flakes respectively. In both cases two configurations are possible: the bottom-bottom $(1 \rightarrow 1)$ and the bottom-top $(1 \rightarrow 2)$, where the ribbon leads are connected to the same $(1 \rightarrow 1)$ or to a different monolayer $(1 \rightarrow 2)$ of the bilayer flake. The bottom-bottom geometry can be achieved by depositing a finite graphene flake onto a graphene nanoribbon, whereas the bottom-top geometry could be realized by the partial overlap of two nanoribbons. ${ }^{24}$ We consider a BGN of width $W$ and length $L$, and mainly focus our study to narrow nanoribbons in the energy range for which only one incident electron channel is active. The bias is applied symmetrically with respect to the top $(-V / 2)$ and the bottom $(V / 2)$ layers.

\section{B. ELECTRONIC STRUCTURE OF THE CONSTITUENTS}

To understand the features of the electronic conductance through these systems, it is necessary to know the band structure of the electrodes and of the central scattering region, so as to identify the channels available for transmission. In this Subsection we give a brief overview of the main features of MGNs and BGNs, indicating the model we have chosen to describe their low-energy properties.

The band structure of graphene has two inequivalent valleys. Within one valley, the low energy properties of 
graphene are well described by the two-dimensional Dirac equation, $H=v_{F} \vec{\sigma} \cdot \vec{p}$, where $v_{F} \sim 1 \times 10^{6} \mathrm{~m} / \mathrm{s}$ is the Fermi velocity, $\vec{p}$ is the momentum operator relative to the Dirac point and $\sigma_{i}$ are the Pauli matrices. The Dirac Hamiltonian, obtained within the $\mathbf{k} \cdot \mathbf{p}$ aproximation, has been applied successfully to the description of the low-energy properties of graphene. For example, it was used by Katsnelson et al. ${ }^{25}$ to predict the Klein paradox in graphene, later confirmed experimentally. ${ }^{26,27}$ The Dirac Hamiltonian acts on a twocomponent spinor, $\left(\phi_{A}, \phi_{B}\right)$, representing the amplitude of the wave function on the two inequivalent triangular sublattices of graphene, labeled $A$ and $B$. The band structure of armchair graphene nanoribbons is obtained from the Dirac equation with the appropriate boundary conditions. ${ }^{4}$ In all nanoribbons the transverse momentum is quantized. For the armchair MGN case, when the number of carbon atoms across the width of the ribbon is equal to $3 m+2$, where $m$ is a positive integer, the smallest transverse momentum is zero. This yields the ribbon metallic with an energy dispersion $v_{F} p_{x}$, where $p_{x}$ is the momentum along the nanoribbon.

In addition to confined states, zigzag MGNs support zero energy surface states located at the edges of the ribbon. ${ }^{3}$ In reciprocal space, surface states occur between the two Dirac cones and their number cannot be described by the two dimensional Dirac equation, which is valid only for the low-energy physics near the cones. Therefore, to describe zigzag graphene nanoribbons we use a nearest-neighbor tightbinding Hamiltonian $H=-t \sum\left(a_{i}^{+} b_{j}+\right.$ h.c. $)$. Here $a_{i}\left(b_{i}\right)$ annihilates an electron on site $i$ of sublattice $A(B)$, and the hopping parameter $t$ is related to the Fermi velocity by $v_{F}=\frac{\sqrt{3}}{2} a t$, where $a$ is the graphene lattice constant, $2.46 \AA$. The tight-binding model has been successfully applied to model the electronic and transport properties of graphene nanoribbons. ${ }^{28,29}$ It captures in a straightforward way the coupling between the unequivalent Fermi points in graphene and gives the same results as the Dirac approximation in the one-valley regime for low energies, where only one propagating mode is available for transport.

Bilayer graphene consists of two coupled graphene layers with inequivalent sites $A_{1}, B_{1}$ and $A_{2}, B_{2}$ on the bottom and top layers, respectively. We consider the Bernal stacking in which the $B_{2}$ sublattice is exactly on top of the sublattice $A_{1}$. Within one valley, the low-energy properties of a biased bilayer graphene are well described ${ }^{30}$ by the Hamiltonian

$H_{B G}=v_{F} \tau_{0} \otimes \vec{\sigma} \cdot \vec{p}+\frac{V}{2} \tau_{z} \otimes \sigma_{0}+\frac{\gamma_{1}}{2}\left(\tau_{x} \otimes \sigma_{x}-\tau_{y} \otimes \sigma_{y}\right)$,

where $\gamma_{1} \sim t / 10$ is the hopping parameter between the closest carbon atoms belonging to different layers, $\sigma_{i}$ are again the Pauli matrices for the sublattice degree of freedom and $\tau_{i}$ are the Pauli matrices for the layer index $\left(\sigma_{0}\right.$ and $\tau_{0}$ are the unit matrices in both subspaces). This Hamiltonian acts on the fourcomponent spinor $\left(\phi_{A}^{1}, \phi_{B}^{1}, \phi_{A}^{2}, \phi_{B}^{2}\right)$ representing the amplitude of the wave function on the two sublattices $A$ and $B$ of the two layers 1 and 2. The energy bands are $\epsilon_{B G}^{2}(p)=v_{F}^{2} p^{2}+\frac{V^{2}}{4}+$ $\frac{\gamma_{1}^{2}}{2} \pm \frac{1}{2} \sqrt{4 v_{F}^{2} p^{2}\left(V^{2}+\gamma_{1}^{2}\right)+\gamma_{1}^{4}}$. The low-energy band has a Mexican hat shape with a minimum gap $\Delta_{1}=\gamma_{1}|V| /\left(\sqrt{\gamma_{1}^{2}+V^{2}}\right)$; see Fig. 1. The minimum gap of the second subband is $\Delta_{2}=$

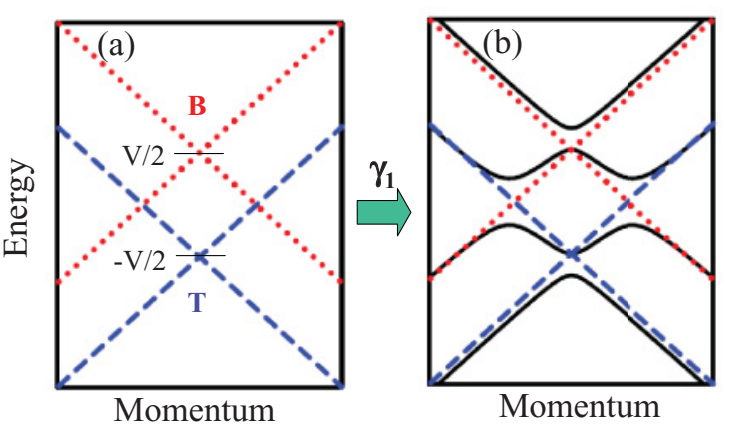

FIG. 1. (Color online) Schematic band structure of a biased graphene bilayer with and without interlayer coupling $\gamma_{1}$. (a) Biased bands without interlayer hopping. A positive (negative) bias $V / 2$ is applied to the bottom (top) layer, producing a rigid shift of the corresponding linear dispersion relation (red dotted lines for the bottom layer, blue dashed lines for the top one). (b) When $\gamma_{1}$ is switched on, gaps open at the band crossings, yielding the well-known Mexican hat shape and split-off bands of the bulk bilayer band structure. This picture allows identifying the top (T)/bottom (B) character of the different branches of the bilayer dispersion relation.

$2 \sqrt{\gamma_{1}^{2}+\left(\frac{V}{2}\right)^{2}}$ and occurs at $p=0$. Figure. 1 illustrates how the Mexican hat shape and the split-off bands arise: without interlayer hopping, the applied bias shifts the linear band dispersions of the two layers; the interaction between layers opens gaps at the intersections of the bands. It also helps us to understand how the electron location changes from the top to the bottom graphene layer, depending on the branch of the dispersion relation.

The electronic structure of an armchair BGN depends on the width of the ribbon. As in the monolayer case, when the number of carbon atoms along a BGN layer is equal to $3 m+2$, the smallest transverse momentum is zero, and the dispersion of the armchair BGN is $\epsilon_{B G}\left(p_{x}\right), p_{x}$ being the momentum along the ribbon; see Fig. 2. In the case of zigzag biased BGNs the system supports two kinds of surface states ${ }^{31}$; (i) states with energies $\sim \pm V / 2$, similar to those occurring in zigzag MGNs, and (ii) valley-polarized states with energies in the gap. At each edge of the ribbon there are two surface states carrying current in opposite directions and belonging to different valleys. These states have a topological nature ${ }^{32}$ but the metallicity of the edge is not protected against intervalley scattering nor against interedge intravalley scattering. The latter occurs when the ribbon width is smaller than the penetration length of the surface states, ${ }^{33} \ell \sim \sqrt{3} \frac{t}{\gamma_{1}} a$, that for realistic values of the interlayer hopping is around $17 a$. This large $\ell$ value produces an interedge scattering gap $\Delta$ in the spectrum of narrow zigzag BGNs; see Fig. 3. Although the valley-polarized surface states can be modeled with the Dirac Hamiltonian, the coupling between states localized in opposite edges is better described using a tight-binding Hamiltonian, which takes into account the coupling between inequivalent Dirac points.

\section{Electronic conductance}

We compute the quantum conductance of the bilayer flakes with monolayer nanoribbon contacts in the coherent transport approximation. Within this assumption, which is not unrealistic for low-dimensional systems and has been realized 

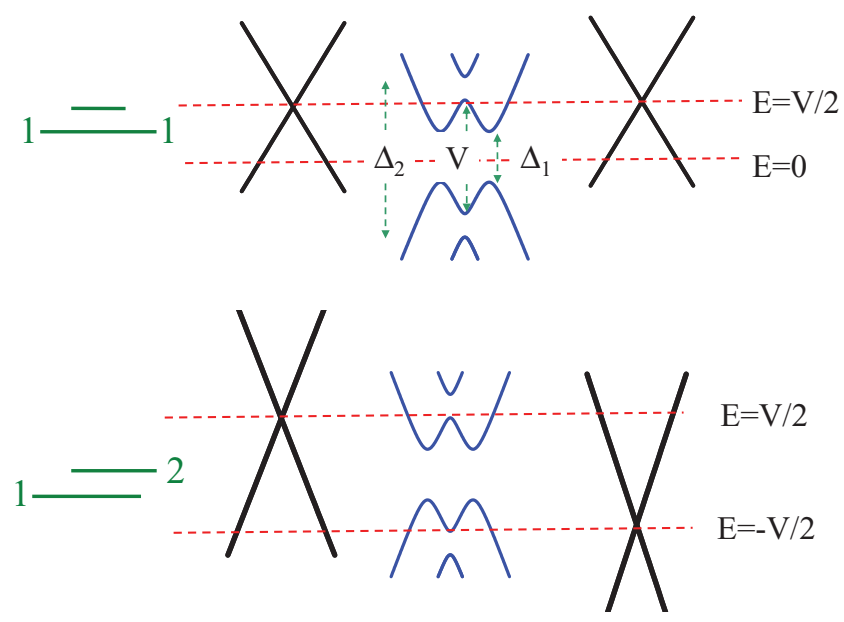

FIG. 2. (Color online) Schematic band structure of the metallic bilayer armchair nanoribbon with monolayer contacts. Upper and lower panels show the bottom-bottom $(1 \rightarrow 1)$ and bottom-top $(1 \rightarrow 2)$ configurations. Left and right dispersions correspond to the metallic monolayer ribbons acting as electrodes, and the central dispersion corresponds to the biased bilayer ribbon. The applied gate bias between bottom and top layers is $V$. For widths for which armchair monolayer ribbons are metallic, the band structure of the bilayer is not affected by the confinement. In the band structure of the bilayer we indicate the three relevant gaps, $V, \Delta_{1}$, and $\Delta_{2}$.

in graphene devices, ${ }^{34}$ the conductance can be computed as the transmission through the system. ${ }^{35}$

In electronic devices, one possible source of scattering can be the metallic contacts that might be needed in the actual experimental system. This is a complex problem, which is currently the focus of substantial research. However, it has been recently shown that with a proper design of the nanoconstriction leading to the nanoribbon, the main contribution to the conductance is due to the nanoribbon device, even with poorly contacting metals. ${ }^{36}$ Another possible source of scattering are the impurities and/or defects that the nanoribbons might have. This point has been theoretically addressed in related systems, showing that even in disordered nanoribbons the transmission in the single-channel regime is almost perfect, because of the absence of backscattering. ${ }^{37}$

In our model, we do not take into account the role of metallic contacts; the electrodes are MGNs, which could be actually used as electrodes in all-graphene nanoelectronics. Thus, for the low-energy range studied in this paper, the ideal system is a good approximation to a realistic system and a necessary starting point for more sophisticated approximations.

\section{Armchair nanoribbons}

As discussed above, the Dirac Hamiltonian describes appropriately the low-energy band structure of armchair nanoribbons. Therefore, we calculate the conductance of such systems, within the single-mode approximation, by matching the eigenfunctions of the Dirac-like Hamiltonian. Given an incident electron coming from the left monolayer ribbon with energy $E$, we compute the transmission coefficient through the bilayer central part to the right monolayer lead. By imposing
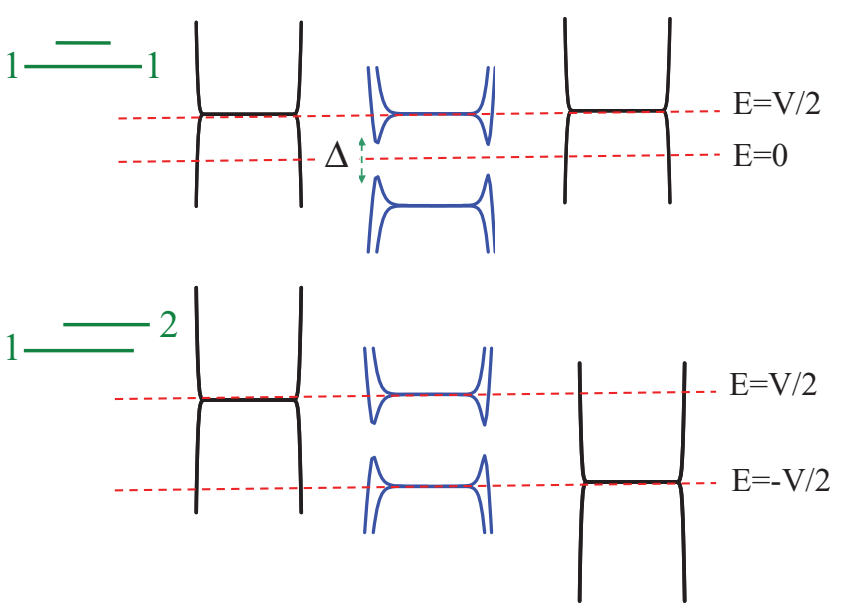

FIG. 3. (Color online) Same as Fig. 2 but for zigzag terminated nanoribbons. For zigzag monolayer nanoribbons, states in different valleys transport charge in opposite directions. In the biased bilayer ribbon, central part, edge localized states with energies $\pm V / 2$ appear, connecting the Dirac points. At the Dirac points of the bilayer ribbons, topological surface states exist in the gap. In wide ribbons these surface states close the energy gap. In narrow bilayer ribbons states in different edges interact and open a gap $\Delta$ in the spectrum.

the appropriate boundary conditions at the ends of the bilayer flake, the transmission, reflection, and the bilayer wave function coefficients can be obtained. In the bottom-bottom configuration $(1 \rightarrow 1)$ the wave functions of the bottom layer $\phi_{A}^{1}$ and $\phi_{B}^{1}$ should be continuous at the beginning $(x=0)$ and at the end $(x=L)$ of the bilayer region. For the top layer the wave function should vanish in one sublattice at $x=0$ and on the other sublattice at $x=L$, e.g., $\phi_{A}^{2}(x=0)=\phi_{B}^{2}(x=L)=0$. In the bottom-top configuration the bottom wave functions $\phi_{\mu}^{1}$ and the top wave functions $\phi_{\mu}^{2}$ should be continuous at $x=0$ and $x=L$, respectively. In addition, the hard-wall condition should be satisfied, $\phi_{B}^{2}(x=0)=\phi_{A}^{1}(x=L)=0$.

From these boundary conditions, the transmission through the bilayer flake is attained. At zero gate voltage the conductance can be obtained analytically ${ }^{24}$; however, the introduction of a gate voltage complicates the analytical expressions of the wave functions and consequently that of the transmission; therefore we compute numerically the conductance.

Finally, for the sake of completeness, we also address the transport properties of armchair ribbons, either metallic or semiconducting, with more than one incoming conducting channel. In this case, the single-mode approximation is not valid, and we employ a tight-binding model, as explained below.

\section{Zigzag nanoribbons}

To describe adequately the low-energy properties of zigzag nanoribbons in the full Brillouin zone it is necessary to use a tight-binding Hamiltonian. In this case we use a Green's function approach to obtain the transport properties. ${ }^{5,35,38}$ To this purpose the system is divided into three parts, specifically, a finite-size bilayer section connected to the right and left 
monolayer semi-infinite leads. The Green's function of the central region is

$$
\mathcal{G}_{C}(E)=\left(E-H_{C}-\Sigma_{L}-\Sigma_{R}\right)^{-1},
$$

where $H_{C}$ is the bilayer Hamiltonian and $\Sigma_{L}$ and $\Sigma_{R}$ are the self-energies at the ends of the bilayer region due to the presence of the leads. These self-energies are numerically calculated employing a decimation algorithm. ${ }^{39}$ The selfenergies contain the information on the type of connection, i.e., $1 \rightarrow 1$ or $1 \rightarrow 2$, of the system. In the linear regime, the conductance is given by

$$
G=2 \frac{e^{2}}{h} T(E)=2 \frac{e^{2}}{h} \operatorname{Tr}\left[\Gamma_{L} \mathcal{G}_{C} \Gamma_{R} \mathcal{G}_{C}^{+}\right],
$$

where $T(E)$ is the transmission at the Fermi energy $E$ and $\Gamma_{L}$ and $\Gamma_{R}$ are the couplings between the bilayer and the left and right monolayer leads respectively.

\section{RESULTS}

\section{A. Metallic armchair nanoribbons in the single-mode regime}

In Fig. 4 we plot the transmission as a function of the incident energy and the applied gate voltage for a metallic armchair nanoribbon system in the $1 \rightarrow 1$ and $1 \rightarrow 2$ configurations. The length of the bilayer flake is $L=35 \sqrt{3} a$ and the value of the interlayer hopping is $\gamma_{1}=t / 10$. The results are independent

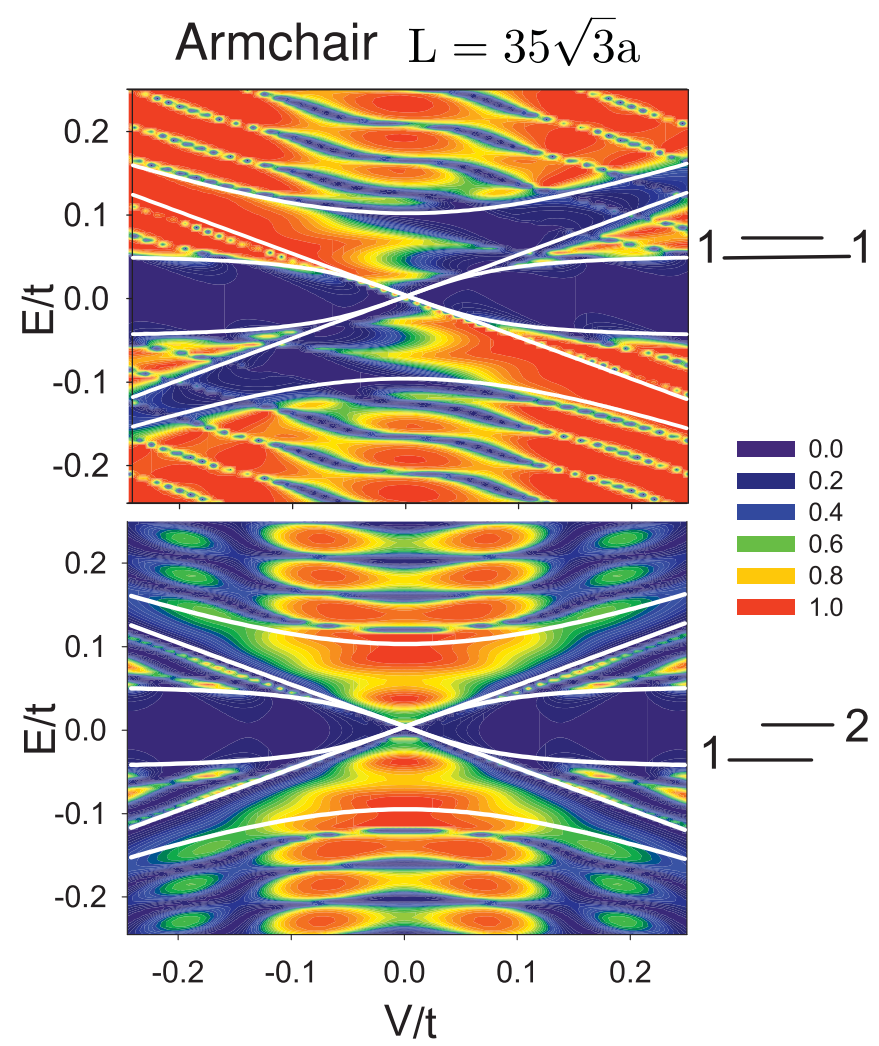

FIG. 4. (Color online) Transmission as a function of energy and applied voltage for metallic armchair nanoribbons in the $1 \rightarrow 1$ and $1 \rightarrow 2$ configuration within the one-mode energy range. The value of the interlayer hopping parameter is $\gamma_{1}=0.1 t$. The length of the bilayer region is $L=35 \sqrt{3} a$. The values of the gap edges $\pm \Delta_{1} / 2$, $\pm \Delta_{2} / 2$, and $\pm V / 2$ are plotted with white lines; see Fig. 2 . of the width of the ribbon, provided that the monolayer ribbons are metallic and that the energy of the incident electron is lower that the energy of the second subband. The transmission is obtained in the continuum approximation, but we have checked that the results coincide with those obtained within the tightbinding approach. Because of the symmetry of the contacts, the $1 \rightarrow 2$ configuration shows electron-hole $(e-h)$ and $V \rightarrow-V$ symmetry. This is not the case for the $1 \rightarrow 1$ configuration, for which the location of the contacts precludes those symmetries. However, this latter configuration presents another symmetry, namely, under the exchange $(e, V) \leftrightarrow(h,-V)$, as can be seen in the top panel of Fig. 4. This can be understood by resorting to the upper part of Fig. 2, which represents the band alignment of monolayer and bilayer graphene armchair nanoribbons for positive $V$. In this case, the crossings of the MGN bands are shifted upward with respect to the center of the bilayer gap. It is easy to see that the monolayer bands would be shifted downward for a negative $V$, thus yielding the aforementioned symmetry under the simultaneous exchange of the sign of the voltage and the carriers.

In both, $1 \rightarrow 1$ and $1 \rightarrow 2$ cases, the conductance is suppressed for energies in the gap $E<\left|\Delta_{1}\right| / 2$, for which there are no available states for the conductance in the bilayer region. We first discuss the results for the bottom-bottom configuration.

In the energy window $\Delta_{1} / 2<|E|<|V| / 2$ there are two propagating states in the bilayer part and the conductance is finite. In the range of energies $|V| / 2<|E|<\Delta_{2} / 2$ there is only one propagating mode in the central region; but in this configuration, when $E$ and $V$ have the same sign, this mode is mostly located in the opposite (top) layer to the leads (bottom), as can be seen in Fig. 1(b), and the conductance is near zero. Thus, by applying a gate voltage between the two layers, we can tune the electronic density in the bilayer. Changing the distribution of carriers from one layer to the other allows to control the conductance of the system by means of an external parameter. For energies $|E|>\Delta_{2} / 2$ the transmission is finite with antiresonances associated with interferences in the bilayer region due to the existence of two propagating channels. ${ }^{24}$ These interferences are weaker for voltages $|V|>\gamma_{1}$, with an overall nonzero conductance, because in this case the incident current from the left electrode is transmitted efficiently to the upper branch of the bilayer dispersion relation, with bottom character, and from there to the right (bottom) lead, with an almost perfect wave-vector matching. ${ }^{40}$ The weak interferences are due to the bilayer-confined states arising from the coupling to the top-layer flake. Note the linear dependence of the position of the antiresonances on the applied voltage: The energy of the confined states in the top layer are displaced by the applied bias $-V / 2$, thus changing the occurence of the antiresonances correspondingly.

In the bottom-top configuration the conductance is not suppressed for $|V| / 2<|E|<\Delta_{2} / 2$ because in this case the incoming and outgoing electrons belong to different layers: The propagating mode in the bilayer has a predominant top character (see Fig. 1), being easily transmitted to the right electrode. For this configuration, the transmission at energies $|E|>\Delta_{2} / 2$ is generally suppressed, even though there are two propagating modes in the bilayer. This can be understood by noticing the wave-vector mismatch ${ }^{40}$ between left and right electrodes produced by the applied bias, as depicted in Fig. 1. 
Away from the gap the transmissions in the $1 \rightarrow 1$ and $1 \rightarrow 2$ configurations are rather complementary; the antiresonances that occur in the $1 \rightarrow 1$ configuration become resonances in the $1 \rightarrow 2$ case. This complementarity of the conductance can be understood by resorting to a simple nonchiral model. Consider an incident carrier, with energy larger than the gap, coming from the left and therefore in the bottom sheet. When arriving at the bilayer central region, the incident wave function decomposes into a combination of the two eigenstates of the biased bilayer. The conductance through the bilayer region is proportional to the probability of finding an electron at the top (bottom) end of the central region for the bottom-top (bottom-bottom) configuration. As the total probability of finding the electron at the end of the bilayer region is unity, the bottom-bottom and the bottom-top transmissions should be the opposite.

\section{B. Zigzag nanoribbons}

In Fig. 5 we plot the transmission as function of the Fermi energy and the applied gate voltage for zigzag nanoribbons in the $1 \rightarrow 1$ and $1 \rightarrow 2$ configurations. These results have been obtained using a tight-binding Hamiltonian and a recursive Green's function technique. 5,35,38 The conductance of zigzag graphene nanoribbons depends on the ribbon width. The results presented in Fig. 5 correspond to a narrow ribbon, $W=11 a / \sqrt{3}$, for which there is only one channel coming

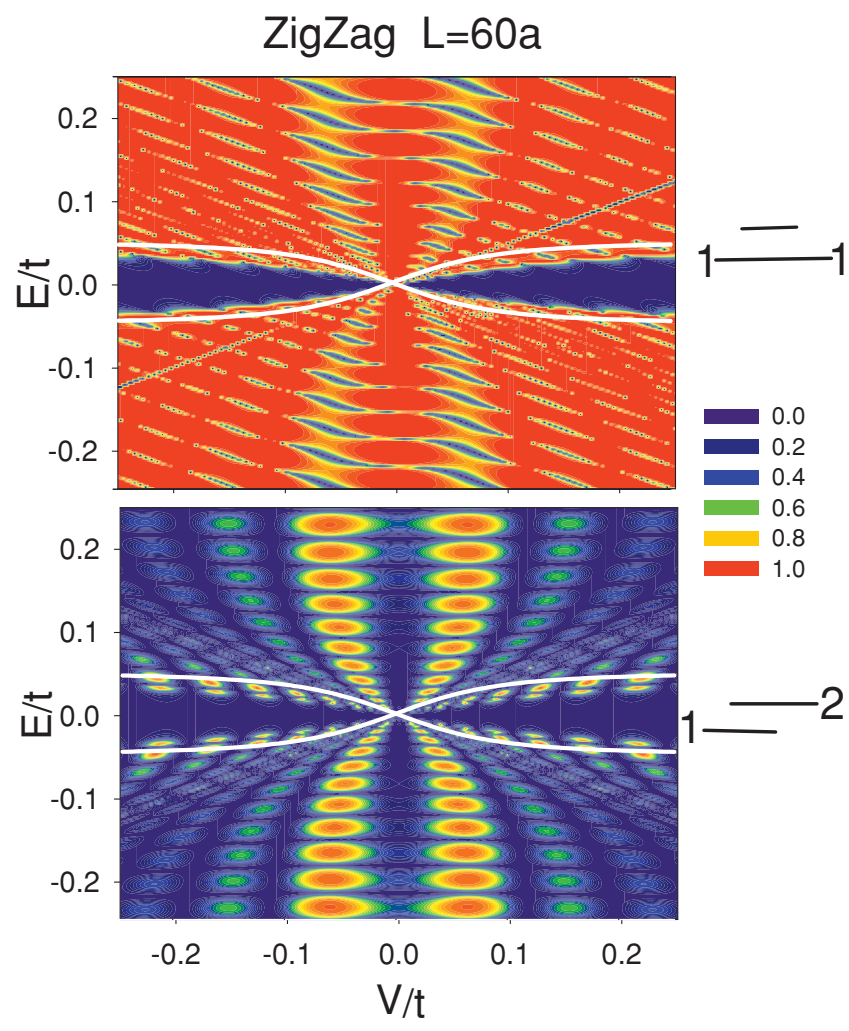

FIG. 5. (Color online) Transmission as a function of energy and applied voltage for zigzag graphene nanoribbons in the $1 \rightarrow 1$ and $1 \rightarrow 2$ configurations. The interlayer hopping parameter is $\gamma_{1}=0.1 t$. The number of atoms across the nanoribbon is 16 , corresponding to a width $W=11 a / \sqrt{3}$. The bilayer region length is $L=60 a$. The values of the gap edges $\pm \Delta_{1} / 2$ are plotted in white lines, see Fig. 2. from the left contact for all the plotted energies. As for the armchair-based systems, there is a strong complementarity between the $1 \rightarrow 1$ and the $1 \rightarrow 2$ configurations, yielding a very different conductance as a function of the energy and bias for the two configurations. Other features, as antiresonances (resonances) in the bottom-bottom (bottom-top) configuration are similar to those occurring in armchair nanoribbons and have the same origin. The same can be said as to the $e-h$ and $V \rightarrow-V$ symmetries for the $1 \rightarrow 1$ geometry and the $(e, V) \leftrightarrow(h,-V)$ symmetry in the $1 \rightarrow 2$ case: They can be explained as for armchair ribbons by considering the relative band alignments depicted in Fig. 3.

In the upper panel of Fig. 5, corresponding to the $1 \rightarrow 1$ configuration, the previous gapped region in the armchair case between $V / 2$ and $\Delta_{2} / 2$ has shrunk to a line of slope $V / 2$. This is easy to understand by observing the zigzag band structure of Fig. 3. Another remarkable feature in Fig. 5 is the existence of a transport gap $\Delta$ smaller than the bulk gap $\Delta_{1}$ of the gated bilayer. As mentioned above, the gap $\Delta$ appears because of the coupling between states with the same valley polarization localized in different edges and moving in opposite directions. The penetration length $\ell$ of these surface states is rather large; for nanoribbons narrower than $\ell$ this produces a noticeable transport gap smaller than the bulk gap.

\section{Nanoribbons with several conducting channels}

We end this section by showing results beyond the singlemode approximation, which we calculate within the tightbinding model. We concentrate in the $1 \rightarrow 1$ geometry, once the differences between the two configurations have been elucidated previously. First, we consider a wider metallic armchair nanoribbon system with $N=23$ dimers across its width, i.e., $W=11 a$, formed by the overlap of a flake of length $L=35 \sqrt{3} a$ over an infinite ribbon. The width was chosen so that the metallic monolayer contacts have, besides the linear bands crossing at $\mathrm{E}=0$, two more parabolic subbands in

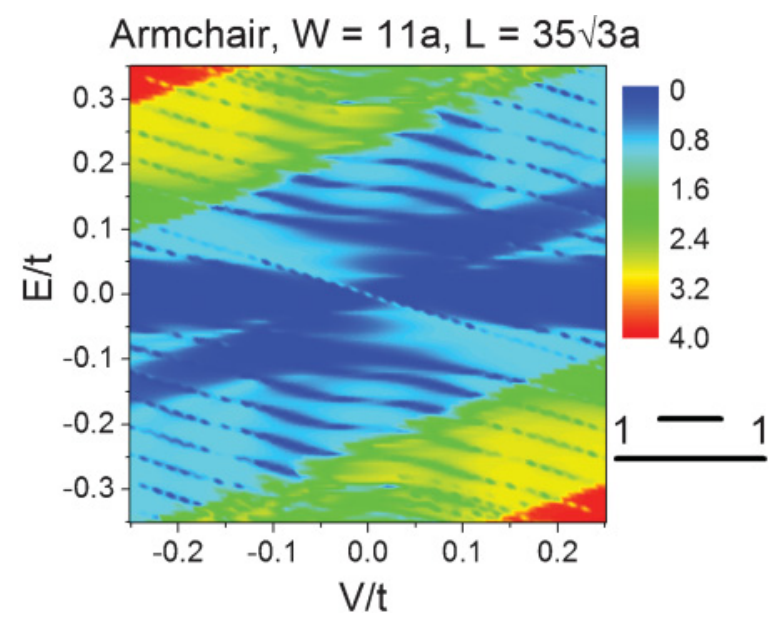

FIG. 6. (Color online) Conductance in units of $2 e^{2} / h$ as a function of energy and applied voltage for an armchair-terminated nanoribbon system in the $1 \rightarrow 1$ configuration. The interlayer hopping parameter is $\gamma_{1}=0.1 t$. The number of dimers across the nanoribbon is 23 , corresponding to a width $W=11 a$. The bilayer region length $L=35 \sqrt{3} a$. 


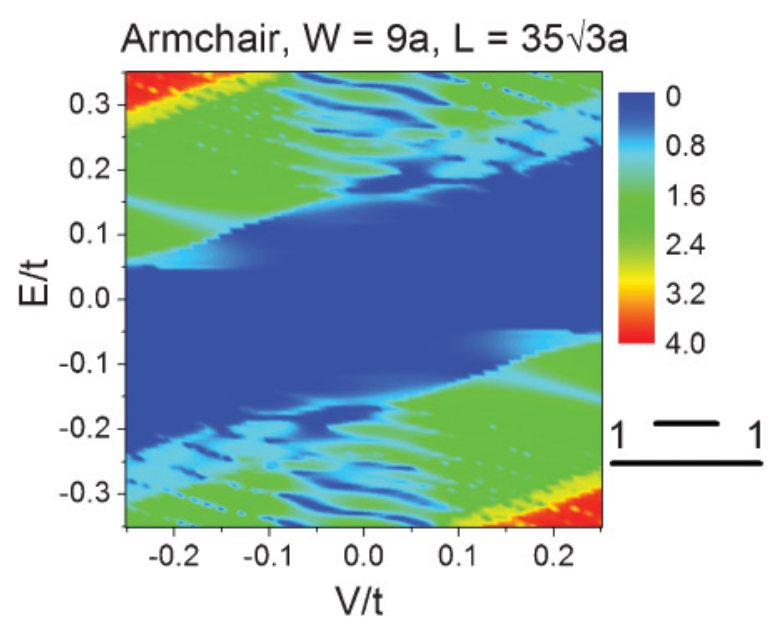

FIG. 7. (Color online) Conductance in units of $2 e^{2} / h$ as a function of energy and applied voltage for a semiconducting armchair-terminated nanoribbon system in the $1 \rightarrow 1$ configuration. The interlayer hopping parameter is $\gamma_{1}=0.1 t$. The number of dimers across the nanoribbon is 19 , corresponding to a width $W=9 a$. The bilayer region length $L=35 \sqrt{3} a$.

the positive energy range explored, with minima at $0.22 \gamma_{0}$ and $0.23 \gamma_{0}$ respectively. These energies mark the threshold for conductance values above 1 and 2 conductance units, as can be seen in Fig. 6. Note that the color scale in this plot varies between 0 and 4 , at variance with the previous transmission results. The main features of the one-channel region are identical to the ones described previously for narrower metallic ribbons, related to the three relevant gaps occurring in the single mode case. For higher energies, larger values of the conductance can be reached, but the overall behavior is similar, with resonances and antiresonances due to the same mechanisms.

Finally, we present results for a system based on the semiconducting armchair ribbon of width equal to $N=19$ ( $W=9 a$ ) and $1 \rightarrow 1$ geometry. As for the previous case, the length of the bilayer portion is $L=35 \sqrt{3} a$. We have chosen a wide enough system in order to have two conducting channels in the energy region studied throughout this work. The gap of the MGN is wider than that of the bilayer, with band edges at $\pm 0.09 \gamma_{0}$. The other two band edges of the monolayer ribbon are at $\pm 0.18 \gamma_{0}$. The monolayer gap sets the threshold for the conductance at $V=0$, as can be seen in Fig. 7, which shows the conductance as a function of energy and voltage bias for this system. In this plot the color scale varies also between 0 and 4 conductance units. For semiconducting systems the monolayer gap dominates the lowenergy region, but resonances and antiresonances as the ones studied for narrower metallic systems can be seen at higher energies.

\section{SUMMARY}

We have calculated the conductance of bilayer graphene flakes with monolayer nanoribbon contacts with a bias voltage between layers. Depending on the position of the electrodes and on the applied bias, there is a strong variation of the conductance. Besides the energy gap opened by the bias, the conductance can be tuned by changing the spatial distribution of the carriers in the bilayer region. Additionally, we have found that the transport gap in bilayer zigzag graphene ribbons is smaller than the bulk bilayer gap. We have atributted this to coupling of edge states with the same valley polarization, which have a rather large penetration length.

Our results show that the transport through graphene bilayer flakes can be controlled by an external parameter.

\section{ACKNOWLEDGMENTS}

This work was partially supported by MEC-Spain under Grant No. FIS2009-08744 and by the CSIC/CONICYT program, Grant No. 2009CL0054. J.W.G. gratefully acknowledges helpful discussions with M. Pacheco, the ICMM-CSIC for their hospitality, and the financial support of MECESUP research internship program, CONICYT (CENAVA, Grant No. ACT27), and USM 110856 internal grant.
${ }^{1}$ L. Liao, Y.-C. Lin, M. Bao, R. Cheng, J. Bai, Y. Liu, Y. Qu, K. L. Wang, Y. Huang, and X. Duan, Nature (London) 467, 305 (2010).

${ }^{2}$ A. H. Castro-Neto, F. Guinea, N. M. R. Peres, K. S. Novoselov, and A. K. Geim, Rev. Mod. Phys. 81, 109 (2009).

${ }^{3}$ K. Nakada, M. Fujita, G. Dresselhaus, and M. S. Dresselhaus, Phys. Rev. B 54, 17954 (1996).

${ }^{4}$ L. Brey and H. A. Fertig, Phys. Rev. B 73, 195408 (2006).

${ }^{5}$ L. Chico, L. X. Benedict, S. G. Louie, and M. L. Cohen, Phys. Rev. B 54, 2600 (1996).

${ }^{6}$ L. Chico, M. P. López Sancho, and M. Muñoz, Phys. Rev. Lett. 81, 1278 (1998).

${ }^{7}$ H. Santos, L. Chico, and L. Brey, Phys. Rev. Lett. 103, 086801 (2009).

${ }^{8}$ J. Nilsson, A. H. Castro Neto, F. Guinea, and N. M. R. Peres, Phys. Rev. B 76, 165416 (2007).
${ }^{9}$ E. V. Castro, K. S. Novoselov, S. V. Morozov, N. M. R. Peres, J. M. B. Lopes dos Santos, J. Nilsson, F. Guinea, A. K. Geim, and

A. H. Castro Neto, Phys. Rev. Lett. 99, 216802 (2007).

${ }^{10}$ I. Snyman and C. W. J. Beenakker, Phys. Rev. B 75, 045322 (2007).

${ }^{11}$ J. B. Oostinga, H. B. Heersche, X. Liu, A. F. Morpurgo, and L. M. K. Vandersypen, Nat. Mater. 7, 151 (2008).

${ }^{12} \mathrm{G}$. Fiori and G. Iannaccone, IEEE Electron Device Lett. 30, 261 (2009).

${ }^{13}$ S. Russo, M. Craciun, M. Yamamoto, S. Tarucha, and A. Morpurgo, New J. Phys. 11, 095018 (2009).

${ }^{14}$ Y. Zhang, T.-T. Tang, C. Girit, Z. Hao, M. C. Martin, A. Zettl, M. F. Crommie, Y. R. Shen, and F. Wang, Nature (London) 459, 820 (2009).

${ }^{15}$ B. N. Szafranek, D. Schall, M. Otto, D. Neumaier, and H. Kurz, Appl. Phys. Lett. 96, 112103 (2010). 
${ }^{16}$ H. Miyazaki, K. Tsukagoshi, A. Kanda, M. Otani, and S. Okada, Nano Lett. 10, 3888 (2010).

${ }^{17}$ X. Li, X. Wang, L. Zhang, S. Lee, and H. Dai, Science 319, 1229 (2008).

${ }^{18}$ D. V. Kosynkin, A. L. Higginbotham, A. Sinitskii, J. R. Lomeda, A. Dimiev, B. K. Price, and J. M. Tour, Nature (london) 458, 872 (2009).

${ }^{19}$ L. Jiao, L. Zhang, X. Wang, G. Diankov, and H. Dai, Nature (London) 458, 877 (2009).

${ }^{20}$ J. Cumings and A. Zettl, Science 289, 602 (2000).

${ }^{21}$ K. Jensen, C. Girit, W. Mickelson, and A. Zettl, Phys. Rev. Lett. 96, 215503 (2006).

${ }^{22}$ J. W. Kang and Q. Jiang, Nanotechnology 18, 095705 (2007).

${ }^{23}$ T. Nakanishi, M. Koshino, and T. Ando, Phys. Rev. B 82, 125428 (2010).

${ }^{24}$ J. W. González, H. Santos, M. Pacheco, L. Chico, and L. Brey, Phys. Rev. B 81, 195406 (2010).

${ }^{25}$ M. I. Katsnelson, K. S. Novoselov, and G. A. K., Nature Phys. 2, 620 (2006).

${ }^{26}$ A. F. Young and P. Kim, Nature Phys. 5, 222 (2009).

${ }^{27}$ N. Stander, B. Huard, and D. Goldhaber-Gordon, Phys. Rev. Lett. 102, 026807 (2009).

${ }^{28}$ H. Zheng, Z. F. Wang, T. Luo, Q. W. Shi, and J. Chen, Phys. Rev. B 75, 165414 (2007).
${ }^{29}$ X. Li, Z. Zhang, and D. Xiao, Phys. Rev. B 81, 195402 (2010).

${ }^{30}$ E. McCann and V. I. Falko, Phys. Rev. Lett. 96, 086805 (2006).

${ }^{31}$ E. V. Castro, N. M. R. Peres, J. M. B. Lopes dos Santos, A. H. Castro Neto, and F. Guinea, Phys. Rev. Lett. 100, 026802 (2008).

${ }^{32}$ I. Martin, Y. M. Blanter, and A. F. Morpurgo, Phys. Rev. Lett. 100, 036804 (2008)

${ }^{33}$ A. Morpurgo, Talk given at the Graphene Week 2010. April 2010, College Park, MD.

${ }^{34}$ C. Berger, Z. Song, X. Wu, N. Brown, C. Naud, D. Mayou, T. Li, J. Hass, A. N. Marchenkov, E. H. Conrad, P. N. First, and W. A. de Heer, Science 312, 1191 (2006).

${ }^{35}$ S. Datta, Electronic Transport in Mesoscopic Systems (Cambridge University Press, Cambridge, 1995)

${ }^{36}$ F. Muñoz-Rojas, J. Fernández-Rossier, L. Brey, and J. J. Palacios, Phys. Rev. B 77, 045301 (2008).

${ }^{37}$ M. Yamamoto, Y. Takane, and K. Wakabayashi, Phys. Rev. B 79, 125421 (2009).

${ }^{38}$ J. González, L. Rosales, and M. Pacheco, Physica B: Condensed Matter 404, 2773 (2009).

${ }^{39}$ M. P. Lopez Sancho, J. M. Lopez Sancho, and J. Rubio, J. Phys. F 14, 1205 (1984).

${ }^{40}$ L. Chico and W. Jaskólski, Phys. Rev. B 69, 085406 (2004). 